\title{
Memories and Futures. Storia e sviluppi di un gruppo di ricerca italiano: dagli studi di efficacia alle analisi delle resistenze al cambiamento
}

\author{
Emilio Fava, ${ }^{1,2}$ Pablo Zuglian, ${ }^{1}$ Dario Ferrario, ${ }^{1,2}$ Daria Taino, ${ }^{1,2}$ \\ Martina Conte, ${ }^{2}$ Francesca Cadeo ${ }^{1}$
}

\section{Sommario}

L'obiettivo di questo articolo è di riassumere brevemente come si sia formato e sviluppato il nostro gruppo di ricerca e quali indirizzi di ricerca abbia preso nel corso degli anni. Tale fine risponde all'esigenza condivisa di una maggiore collaborazione e integrazione tra i vari gruppi che si sviluppi anche al di fuori dei contatti stimolanti, ma purtroppo sporadici, che avvengono ai congressi, sia nazionali che internazionali, in modo da aprire eventuali nuove strade di condivisione di percorsi di ricerca comune. Siamo convinti che la conoscenza reciproca sia la "via regia" alla costruzione di nuovi progetti e alla realizzazione di quelle conoscenze scientifiche per cui la nostra Società è nata.

\section{Parole chiave}

Efficacia dei trattamenti, relazione terapeutica, diagnosi psicodinamica, resistenze al cambiamento, diagnosi dimensionale, formazione

1 Università degli Studi di Milano - S.C. Psichiatria 4, A.O. Niguarda Ca' Granda, Milano. ${ }^{2}$ Associazione Gruppo Zoe, per la formazione e lo studio su la qualità e l'efficacia delle cure psichiche, Milano.

Referente: Emilio Fava

Corrispondenza: Emilio Fava

E-mail: emilio.fava@unimi.it 


\section{Introduzione}

Il gruppo milanese SPR nacque nel 1987, presso l'Istituto di Clinica Psichiatrica dell' Università degli Studi di Milano a opera di Salvatore Freni ed Emilio Fava. Furono presi contatti con i gruppi di ricerca stranieri e in particolare con il gruppo di Ulm (Thöma e Kachele) e con il gruppo di Luborsky negli Stati Uniti. Iniziò così un lavoro di traduzione e diffusione di alcuni testi base per la ricerca come ad esempio il "Trattato di terapia psicoanalitica" (Thöma \& Kachele, 1985), il "Manuale di psicoterapia supportivo-espressiva" (Luborsky, 1984) e "Capire il transfert" (Luborsky \& Crits-Cristoph, 1990), manuale del metodo CCRT di cui venne valutata l'affidabilità nel contesto italiano. In quel periodo iniziò il lavoro di traduzione e validazione di alcune scale di valutazione multidimensionale degli esiti come la HSRS (Luborsky, 1962; Freni, Azzone, Gaburri, \& Gigli, 1998), dell'alleanza di lavoro come l'HA (Luborsky, 1976; Verga, 1999) e dei meccanismi di difesa (Perry, 1991), oltre all'utilizzo del metodo CCRT per la prima volta. L'obiettivo principale del gruppo era quello di sperimentare la possibilità di operazionalizzare alcuni costrutti fondamentali del pensiero psicoanalitico, e di individuarne le potenzialità euristiche e gli ambiti di applicazione, in un contesto tendenzialmente diffidente se non ostile.

Il lavoro svolto in quegli anni, in collaborazione con i vari gruppi di ricerca nati nel frattempo in Italia, portò all'organizzazione del primo congresso di SPR Italia a Cernobbio nel 1996 e all'elezione di Salvatore Freni come primo presidente della sezione italiana della Società di Ricerca in Psicoterapia e direttore della rivista Ricerca in Psicoterapia (1998).

Nel 1994 si costituì presso la Clinica Psichiatrica dell'Università degli Studi di Milano ad Affori un secondo gruppo, coordinato da Emilio Fava e Cinzia Masserini, che pur continuando le ricerche sul processo 
terapeutico utilizzando i metodi CCRT (Fava, 1995; Masserini, Fava, Arduini, Borghetti, Calini, Corbellini, Ferri, Fontolan, Gatta, Mazzariol, Pazzi, Spoletini, \& Pazzaglia, 1998) e SASB (Benjamin, 1974; Capelli, Fava, Taglietti, Aliprandi, Arduini, Freni, Schadee, \& Vigorelli, 2005; Fava \& Vigorelli, 2006), iniziò a occuparsi anche di studi di efficacia, utilizzando metodi quantitativi e qualitativi. Questi studi hanno portato alla pubblicazione di alcuni articoli riguardanti l'esito delle psicoterapie (Fava, Pazzi, Arduini, Masserini, Lammoglia, Lomazzi, Landra, Pazzaglia, \& Carta, 2000; Osimo, Merlo, Arduini, Landra, Fava, Masserini, Carta, \& Pazzaglia, 1998; Rozzi, Rozzi, Fava, Lazzari, Baruhk, \& Fontolan, 1997; Ferrari, Pinzi, Camarda, \& Roustayan, 2005), le interruzioni non concordate di trattamento (Masserini et al., 1998; Fava, Masserini, Borghetti, Camarda, Fontolan, \& Duca, 2001) e la formazione degli psichiatri e il loro punto vista sulla cura nei servizi pubblici di salute mentale (Ba, Fava, \& Carta, 1992; Fava, 2004; Lomazzi, Fava, Landra, D’Angelo, Lammoglia, Pazzi, Calini, Arduini, Barattini, \& Carta, 1997) confluiti poi nel libro "Efficacia delle psicoterapie nel servizio pubblico" (Fava \& Masserini, 2002) e in successivi lavori (Fava \& Masserini, 2006). Gli studi sull'efficacia dei trattamenti rispondevano alla domanda su quali trattamenti andassero praticati nei Servizi Pubblici, in un contesto in cui si imponeva una loro razionalizzazione sulla base di dati sul'evidenza dei risultati terapeutici. Questo orientamento, nel contesto di una esigenza di integrazione degli interventi da attuare nei servizi psichiatrici e nella pratica clinica, rimane uno dei principali punti di interesse del gruppo della Clinica Psichiatrica Universitaria.

La ricerca sugli esiti dei trattamenti ha portato, nel 2000, alla collaborazione con il gruppo di Marta Vigorelli (Università di MilanoBicocca), che si stava occupando di valutazione dell'efficacia nei Servizi Pubblici (utilizzando il metodo HONoS) rispondendo alla richiesta di 
valutazione di qualità dei servizi che stava emergendo in quel periodo e che si è sempre più sentita nei servizi di salute mentale in Italia.

Il problema della valutazione degli esiti e della valutazione della qualità dei trattamenti implica lo studio delle cause dei fallimenti terapeutici anche nei casi di trattamenti di provata efficacia. Per questo è nata anche una collaborazione con il gruppo Bicocca volta allo studio dell'andamento della relazione terapeutica in pazienti poor outcome utilizzando il metodo SASB. Inoltre è stato sviluppato e applicato un sistema di valutazione della qualità dei trattamenti utilizzando alcuni strumenti utilizzati per l'analisi dei single-case (ESMS).

Dal 2004, sempre all'interno della Clinica Psichiatria Universitaria S.C. Psichiatria 4 A.O. Niguarda, si è sviluppato un nuovo filone di studio orientato alla diagnostica psicodinamica che partendo dalle ricerche sulla OPD (Diagnosi Psicodinamica Operazionalizzata; OPD Task Force, 1996, 2006) si muove verso lo studio della dimensionalità nella diagnostica psicodinamica, per comprendere i processi psicoterapeutici e permettere la personalizzazione dei trattamenti, fattore di provata efficacia. Dal 2007 nasce la collaborazione con M. Cierpka di Heidelberg ed il gruppo di lavoro OPD internazionale, che ha portato al lavoro di traduzione e di cura dell'edizione italiana della seconda versione del manuale OPD, trasformato in manuale per la diagnosi e la pianificazione del trattamento.

Dal gruppo di ricerca su OPD si è costituita, allargandosi anche ad altri progetti, nel 2009 l'Associazione "Gruppo Zoe - per la formazione e lo studio sulla qualità e l'efficacia delle cure psichiche" che si sta occupando delle applicazioni cliniche dei risultati ottenuti dalla ricerca empirica in psicoterapia.

Attualmente è nato un gruppo di lavoro in collaborazione con la Sigmund Freud University di Vienna (O. Gelo) per un'analisi qualitativa dei colloqui diagnostici, usando le interviste OPD raccolte in Italia. 


\section{Linee di ricerca}

\section{Relazione terapeutica, resistenze ed esito dei trattamenti ${ }^{1}$}

Il problema delle resistenze al cambiamento, anche nel caso di terapie di provata efficacia, è una delle frontiere più attuali della ricerca (Benjamin, 2004; Beutler, Moleiro, \& Taleb, 2001; Beutler, Rocco, Moleiro, \& Talebi, 2001; Beutler, Harwood, Michelson, Song, \& Holman, 2011; Castonguay \& Beutler, 2005). Inoltre è stata posta come priorità per la ricerca (Norcross, 2001) la necessità di comprendere come i fattori terapeutici di provata efficacia interagiscano con i meccanismi che regolano il cambiamento terapeutico.

Il termine resistenza applicato al comportamento del paziente implica la difficoltà o il rifiuto di cooperare o di cambiare. Essa è stata considerata sia uno stato, che un tratto durevole. In psicoanalisi il termine resistenza veniva considerato l'espressione di una lotta interna difensiva contro la sofferenza o l'angoscia, che indirettamente rallentava il lavoro di comprensione/interpretazione, cui veniva attribuito il ruolo di fattore terapeutico principale. In psicologia sociale lo stesso termine veniva definito come "condizione della mente attivata da una minaccia alla propria libertà, che motiva l'individuo a ripristinare la libertà minacciata" (Brehm \& Brehm, 1981). In generale la ricerca afferma che la resistenza del paziente, indipendentemente dalle sue origini, impedisce il raggiungimento degli obiettivi terapeutici (Beutler, Clarkin, \& Bongar, 2000) e gli psicoterapeuti dovrebbero essere incoraggiati a produrre meno resistenza possibile.

\footnotetext{
${ }^{1}$ Gruppo costituito da D. Ferraio, D. Taino, E. Fava, F. Cadeo, H. Schadee, M. Conte, G. Sasso.
} 
Secondo una prospettiva recente che coniuga la dimensione clinica della teoria dell'attaccamento e la teoria intersoggettiva (Wallin, 2007), la resistenza avrebbe quasi sempre anche un significato interpersonale e potrebbe essere vista come il risultato della collusione tra paziente e terapeuta per assicurarsi che non accada nulla di nuovo o di minaccioso. Detto in modo diverso, la resistenza del paziente all'esperienza - esperienza di ciò che si percepisce come sofferenza emotiva insopportabile - sarebbe collegata alla paura di scarso aiuto da parte del terapeuta (sfiducia). La proposta è allora quella di vedere la resistenza come una comunicazione su aspetti dell'esperienza del paziente difficili da tollerare e difficili da tradurre in parole. In questa prospettiva, i pazienti cercherebbero di trasmettere in modo indiretto o di evocare nel terapeuta, ciò che da soli non riescono a sopportare.

Noi abbiamo considerato la relazione interpersonale reale, nell'accezione di Gelso, come variabile d' efficacia, e i processi introiettivi, intesi come processi che permettono l'acquisizione di nuove rappresentazioni e capacità in seguito al trattamento, come meccanismo di cambiamento.

Per relazione reale si intende un rapporto realistico e non deformato, ma piuttosto caratterizzato dall'incontro di due persone nella realtà attuale, la quale implica la partecipazione di entrambe e il riconoscimento che ognuna viene modificata dall'altra all'interno di questo processo (Gelso, 2002). Gelso $(2004,2009)$ propone inoltre di definirla come costituita da due elementi: realismo e genuinità, dove il realismo riguarda l'esperienza dell'altro (il terapeuta) in quanto persona reale, e la genuinità la capacità del terapeuta di essere veramente quello che è, contrapposto all'essere falso o inautentico. Gelso propone due ulteriori dimensioni (sub-elementi): la valenza della relazione interpersonale (positività versus negatività) e la magnitudo, cioè l'ampiezza della relazione reale che esiste. In ultima analisi la relazione 
Ricerca in Psicoterapia / Research in Psychotherapy 2010; 2 (13): 53-77 http://www.researchinpsychotherapy.net

reale, in questa concezione, ha poco a che fare sia con l'alleanza di lavoro che con gli aspetti difensivi e proiettivi del paziente sul terapeuta e viceversa (transfert e controtransfert). Essa inizia al momento del primo contatto e opera silenziosamente per tutta la durata della terapia.

Dati gli obiettivi prefissati si è deciso di utilizzare il modello SASB (Structural Analysis of Social Behavior; Benjamin, 1974, 1993) in quanto uno dei più sofisticati strumenti che consentono di operazionalizzare questi costrutti (Benjamin, 2004; Fava \& Vigorelli, 2006). Tale metodo permette infatti di valutare le transazioni comunicative tra paziente e terapeuta in sedute registrate, ma anche di valutare i moti relazionali verso il sé, cioè come l'individuo tratta se stesso, nella evoluzione della terapia (introiezione di nuove capacità e attitudini).

E stata quindi necessaria una prima fase di validazione del modello e di addestramento al metodo tra i ricercatori, fino al raggiungimento di una buona affidabilità intergiudici e intragiudice $(0.72<\mathrm{k}<0.88)$, ripetuta più volte nel corso del programma di ricerca.

Sono stati analizzati i trascritti di sedute appartenenti a 10 coppie di pazienti (con stessa diagnosi DSM-IV-TR, APA, 2000), ciascuna delle quali trattata dallo stesso terapeuta, con esiti opposti (valutati a 0, 6 e 12 mesi attraverso la somministrazione dell'SCL-90-R e di una scala Likert compilata dal clinico): dieci soggetti ad esito positivo, e dieci soggetti ad esito insoddisfacente, (che hanno beneficiato poco o per nulla del trattamento psicoterapeutico). Tutti i soggetti inclusi nello studio hanno completato almeno un anno di terapia a differente orientamento (a orientamento psicoanalitico o cognitivo), a una seduta la settimana. Per ogni paziente sono state valutate nel primo anno di psicoterapia due sedute a 0, 4, 8 e 12 mesi; gli unici criteri di esclusione erano l'età inferiore ai 18 anni e la presenza di ritardo mentale. 
Il lavoro di ricerca si è focalizzato sulla valutazione del legame tra il processo terapeutico, valutato in termini di dinamiche interpersonali tra terapeuta e paziente, e l'esito dei trattamenti al fine di evidenziare eventuali differenze tra $\mathrm{i}$ trattamenti a esito positivo e quelli a esito insoddisfacente. Più in dettaglio il primo obiettivo della ricerca è stato quello di individuare specifici stili interpersonali (che definiscono l'atteggiamento relazionale dei terapeuti e dei pazienti) nei due gruppi d'esito e ricostruire le dinamiche processuali che si verificano tra i membri della coppia. Sono stati così individuati dei "moti problematici"2, caratterizzati da un atteggiamento interpersonale di aggressività e controllo benevolo o neutro per quanto riguarda il terapeuta e risposte avversive di aggressività, sottomissione neutra e ostile o evitamento, da parte del paziente.

Ciò è risultato essere in linea con quanto evidenziato in letteratura (Henry, 1996; Henry, Schacht, \& Strupp, 1986; Henry \& Schacht, 1994; Henry \& Strupp, 1994; Hilliard, Henry, \& Strupp, 2000; Nelson, 2004), rispetto al potere predittivo del livello di ostilità/aggressività sull'esito delle psicoterapie. Nel nostro campione, pur evidenziandosi un minor numero di moti caratterizzati da ostilità, la differenza fra i due gruppi d'esito è statisticamente significativa.

Alcuni studi hanno rilevato un ruolo tendenzialmente controproducente di una modalità terapeutica di intervento direttiva, individuando come fattore discriminante tra terapie a esito differente una scarsa alleanza terapeutica e a un alto livello di controllo da parte

2 Con la definizione "moti problematici" si fa riferimento a quei movimenti interpersonali sia del terapeuta che del paziente caratterizzati da ostilità positiva e livelli di controllo variabile (rose 6, 7 e 8 del modello SASB) e a quelli posti alle polarità estreme dell'asse Interdipendenza (rose 1 e 5 del modello). L'insieme di questi movimenti interpersonali di tipo intransitivo è stato definito "moti problematici" in quanto precedenti ricerche (Alpher, 1991; Henry, 1996; Henry et al., 1986; Henry \& Strupp, 1994; Hilliard et al., 2000; Nelson, 2004), hanno evidenziato come essi siano prognostici di esiti negativi. 
61

Ricerca in Psicoterapia / Research in Psychotherapy 2010; 2 (13): 53-77 http://www.researchinpsychotherapy.net

del terapeuta (Svartberg \& Stiles, 1991; Horvath \& Greenberg, 1994; Hilliard et al., 2000; Horvath, 2009).

Una differenza, rispetto a questi dati, è che nel nostro campione i livelli di controllo da parte del terapeuta non differenziano le terapie good-outcome da quelle poor-outcome, a un primo livello di analisi descrittivo (analisi delle frequenze). Si è quindi proceduto con un' analisi sequenziale, a due transizioni, per valutare in quale misura l'intervento del terapeuta determini la risposta del paziente e viceversa, in linea con la recente pubblicazione di Von der Lippe (Von der Lippe, Monsen, Rønnestad, \& Eilertsen, 2008). La domanda clinica in questo senso è "che cosa ha fatto scattare la risposta avversiva e quale forma essa ha preso: antagonismo, ritiro o tutte e due?". I primi risultati (Capelli et al., 2005; Marchesi, Vigorelli, Schadee, Fava, \& Capelli, 2007), si sono avvalsi di tre delle dieci coppie del campione complessivo e hanno evidenziato come modalità relazionali di ritiro, resistenza o difficoltà a seguire il terapeuta inducano in quest'ultimo l'incremento di un comportamento controllante. Sul campione complessivo le analisi confermano questa modalità interpersonale e mostrano come nel gruppo delle terapie a esito insoddisfacente, il terapeuta gestisca i moti connotati da sottomissione ostile con percentuali significativamente maggiori di controllo biasimante, assenti invece nel gruppo a esito positivo. I moti di presa di distanza inducono inoltre nel clinico un incremento sempre maggiore di dosi di controllo sia dal punto di vista quantitativo che del livello di ostilità. Parallelamente, appare come i moti di controllo, anche benevoli, da parte del terapeuta stimolino nei pazienti poor outcome livelli di resistenza maggiori, individuabili nei moti di presa di distanza, sottomissione ostile e inversione del focus relazionale.

La ricerca fin qui condotta ci ha portato ad avere delle informazioni abbastanza precise su quali stili e quali dinamiche interpersonali 
caratterizzano le terapie a esito insoddisfacente; il gruppo si è quindi domandato: "cosa può aver facilitato o ostacolato il cambiamento?". La nostra attenzione si è così focalizzata sullo studio della reciproca influenza tra la relazione reale e i meccanismi del cambiamento, in particolare tra la resistenza alla terapia e i meccanismi di tipo introiettivo. Riferendoci alla tradizione psicoanalitica, abbiamo considerato con il termine introiezione l'apprendimento di nuove modalità di rappresentazione, di gestione di sé e della relazione con l'altro. La ricerca in questo campo ha dimostrato che l'interiorizzazione in strutture intrapsichiche del processo interpersonale che si attua in terapia è correlato all'esito dell'intervento (Fossi, 2003).

Questa ultima fase del nostro lavoro si è quindi concentrata sulla valutazione degli introietti ${ }^{3}$, attraverso una analisi statistica descrittiva delle frequenze e del loro andamento nel tempo. I risultati evidenziano come la qualità e l'andamento nel tempo degli introietti siano considerevolmente e precocemente, nell'anno considerato, differenti nei due gruppi di esito. I pazienti a esito positivo mostrano un incremento della capacità di prendersi cura di sé e una diminuzione di modalità biasimanti e aggressive verso il sé, i pazienti a esito insoddisfacente mostrano, invece, un livello elevato e stabile nel tempo di moti autobiasimanti e trascuranti e assenza di moti di accudimento di sé nell'arco di tempo considerato. Tali risultati sono statisticamente significativi nelle analisi caso per caso, per diciannove pazienti su venti, dimostrando un alto valore predittivo.

L'esperienza con un terapeuta che "si prende cura" e le successive possibili introiezioni, sembrano quindi facilitare nel paziente quel processo di cambiamento che si verifica nelle terapia a esito positivo; questo invece non si verifica, perlomeno nei tempi considerati dalla ricerca, nei casi a esito più sfavorevole. In questi casi precocemente è

\footnotetext{
${ }^{3}$ Per un ulteriore approfondimento del concetto di introietto, e le modalità con cui esso viene valutato nel colloquio clinico, si rimanda ai lavori di Benjamin $(2004,2005)$.
} 
individuabile un atteggiamento "evitante" da parte del paziente non indotto dal terapeuta, ma che induce nel terapeuta atteggiamenti controllanti sia benevoli che neutri e talvolta aggressivi. Questo atteggiamento tende a modificarsi poco nel primo anno di terapia e sembra inibire i processi introiettivi di cambiamento. Le prospettive di questo filone di ricerca riguardano ora le possibili strategie per superare questo handicap.

Sempre nella prospettiva di individuare come funzionano i meccanismi collegati ai cambiamenti terapeutici e le possibili forme di resistenza, stiamo sviluppando uno strumento che permetta di valutare le oscillazioni introiettive e proiettive che caratterizzano gli scambi interpersonali e i loro livelli di complementarietà, sul modello della Infant Research.

Il modello VERA (Verbal and Enactive Representations Analysis) che operazionalizza le concezioni teoriche di G. Sasso (Sasso, 2009), si propone come "ponte" tra Infant Research e Psicoanalisi, e permette di avanzare alcune ipotesi neurologiche su come si regoli nel cervello del bambino l'elaborazione dell'interazione materna, su come la madre possa modificarla e su come questo processo si replichi nell'interazione tra paziente e terapeuta.

Si parte dalla constatazione che, alla nascita, sono già mature alcune zone cerebrali che assicurano la comunicazione tra il cervello e l'ambiente. Il bambino si organizza assumendo, dunque, come vertice il proprio organismo e avendo come fondamento l'esperienza propriocettiva e senso-motoria, che rimane pertanto a sostegno della successiva integrazione sottocorticale-corticale. Emerge, qui, l'attività di un complesso sistema senso-motorio.

Il presupposto di VERA è che il processo di sintonizzazione possa formarsi proprio perché i sistemi nervosi del bambino e della madre posseggono una "oscillazione autonoma" fronto-occipitale, le cui 
caratteristiche intrinseche permettono l'inaugurarsi e la regolazione della sintonizzazione. Gradualmente, infatti, l'oscillazione produce opportuni flussi nervosi che vanno dalle aree sensoriali-percettive verso le aree motorie, in modo da vincolare i pattern sensoriali in sviluppo alle corrispondenti risposte motorie e, viceversa, produce flussi dalle aree motorie verso quelle sensoriali, affinché i programmi motori innati possano vincolarsi alle informazioni percettive necessarie per riconoscere e interagire con l'oggetto materno.

Chiaramente, la dinamica, dopo la nascita, non è più autonoma ma viene influenzata dall'apporto sensoriale materno.

Assumendo inoltre (Sasso, 2009) che il linguaggio sia una specializzazione del reticolo senso-motorio e dunque che vi sia una continuità tra il dominio implicito e quello riflessivo-verbale accomunati dalla intenzionalità comunicativa, lo strumento VERA è in grado di cogliere il contesto non verbale del linguaggio (BCPSG, 2008) a partire dai trascritti delle sedute e cogliere "i micro-cambiamenti momento per momento dell'interazione transfert-controtransfert", in particolare negli enactment, a partire dalla valutazione delle narrative (rappresentazioni verbali) e dalla dinamica comunicativa Proiettiva-Introiettiva terapeutapaziente (enactive representations).

\section{Sviluppo della diagnostica dimensionale e OPD $2^{4}$}

Provenendo da una Clinica Psichiatrica Universitaria, luogo in cui si cerca di coniugare efficacemente la ricerca con la formazione e l'attività clinica, questo gruppo è nato dall'esigenza di utilizzare una metodologia diagnostica che fosse più vicina alla realtà clinica del paziente che ci

\footnotetext{
${ }^{4}$ Gruppo costituito da P. Zuglian, M. Magni, E. Fava, M.L. Zuccarino, D. Ferrario, A. Testa, M.E. Pagliari, M. Greco, F. Cadeo, T. Monea, G. Mentasti, D. Taino, L. Primerano, L. Varischio, P. Cafagna, P. Bondi, S. Crispino.
} 
troviamo di fronte piuttosto che alla ateoreticità della diagnostica nosografico-descrittiva psichiatrica classica.

La scelta di utilizzare come sistema diagnostico OPD (Diagnosi Psicodinamica Operazionalizzata; OPD Task Force, 1996), e in seguito la possibilità di poter tradurre e curare l'edizione italiana della seconda versione di OPD (OPD-2; OPD Task Force, 2006), è avvenuta per il suddetto motivo. Rispondeva, quindi, alle nostre esigenze cliniche e, come abbiamo scoperto meglio in seguito, anche alle esigenze di formazione nell'ambito della psicoterapia e della clinica psicodinamica (la valutazione dei conflitti intrapsichici, del livello di interazione strutturale, dei pattern relazionali disfunzionali ripetitivi).

OPD-2 è uno strumento diagnostico multiassiale di matrice psicodinamica e con numerosi riferimenti anche alle teorie cognitive e ai risultati della ricerca empirica in psicoterapia. È costituito da 5 assi che nomineremo brevemente (per una descrizione più completa si veda Zuglian, Papini, Conte, Fava, \& Ferrari, in press):

1. esperienza di malattia e presupposti per il trattamento;

2. relazioni interpersonali;

3. conflitti intrapsichici;

4. struttura;

5. disturbi mentali e psicosomatici.

Parallelamente abbiamo imparato come questo strumento potesse essere utilizzato anche per fornire una diagnostica dimensionale in grado di evidenziare dei fattori comuni tra le variabili che potessero fornirci ancora più agilmente delle informazioni diagnostiche, quindi in qualche modo riassuntive, del funzionamento del paziente anche in senso trasversale agli assi dello strumento OPD (possibilità quindi di una doppia lettura dello strumento sia in senso longitudinale, asse per asse, che trasversale, interasse). 
66

Ricerca in Psicoterapia / Research in Psychotherapy 2010; 2 (13): 53-77 http://www.researchinpsychotherapy.net

Le prime ricerche (Conte, Ferrari, Fava, Papini, Zuglian, Tajani, Fiorina, Magni, Maramieri, Primerano, \& Freni, 2007; Ferrari, Papini, Zuglian, Conte, Fava, Tajani, Fiorina, Magni, Maramieri, Primerano, \& Freni, 2007) si sono quindi mosse per osservare le possibili dimensioni di funzionamento OPD in un campione di pazienti psichiatrici, eterogeneo per diagnosi sia in Asse I che in Asse II del DSM-IV TR. In seguito a quei risultati preliminari, abbiamo deciso di occuparci specificamente di un campione di pazienti omogeneo per diagnosi in Asse I, ma estremamente eterogeneo per decorso, risposta ai trattamenti e prognosi, in cui dalla letteratura non emergono dei trattamenti efficaci secondo gli attuali standard della ricerca, cosa in realtà molto diversa dall'esperienza dei clinici, di vari orientamenti, che si occupano specificamente di questi disturbi. La scelta è così caduta sui Disturbi del Comportamento Alimentare (DCA), disturbi caratterizzati da un elevatissimo tasso di crossover diagnostico tra i disturbi (Anoressia Nervosa e Bulimia Nervosa) e anche tra i sottotipi diagnostici (Restrittivi e con Condotte di eliminazione), che arriva secondo alcune ricerche fino al 65\%. Questo dato ci indica la necessità di un assessment diagnostico che sia differente da quello attualmente in uso (che viene giudicato inadeguato anche da coloro che l'hanno formulato e che stanno purtroppo riproponendo anche nella V edizione del DSM, APA DSM-V Development, on line).

Abbiamo scelto un campione di pazienti afferenti a un servizio di nutrizione clinica che usualmente non prevede una valutazione e un percorso psicoterapico. Questo è rilevante perché il campione risulta così maggiormente variegato dal punto di vista delle idee alla base della malattia e delle motivazioni alla cura (Asse I OPD). Ogni dimensione, attraverso il calcolo dei factor score derivanti dall'analisi fattoriale, può essere successivamente valutata sul singolo caso permettendoci di studiare attentamente l'esito e il follow-up di queste pazienti, le quali vengono indirizzate a differenti trattamenti sulla base del giudizio 
clinico e permettendoci in tal modo di mantenere la struttura naturalistica dello studio. Le dimensioni deriveranno da un'analisi fattoriale che sarà effettuata su 300 casi, numero minimo per avere dei risultati che siano robusti dal punto di vista della significatività statistica. Attualmente i risultati preliminari derivano da un'analisi effettuata su di un campione decisamente meno numeroso, 50 casi, ma attraverso l'utilizzo del metodo dei fattori principali abbiamo cercato di aumentare la significatività di tali dati in quanto abbiamo eliminato la varianza intergruppo ed esaminato solamente quella intragruppo. L'analisi fattoriale viene quindi effettuata prima sull'Asse I di OPD in modo da trovare le dimensioni inerenti la personalizzazione (Lambert, 2001; Fava, Zuglian, Taino, \& Di Genova, 2009) del trattamento (indicazioni e costruzione dell'alleanza terapeutica) e successivamente viene effettuata una nuova analisi tra queste dimensioni trovate e gli assi dei conflitti intrapsichici e della struttura.

In seguito alla valutazione dell'esito e del follow-up cercheremo, attraverso una regressione multipla, di valutare l'esistenza di eventuali predittori di esito sia sotto forma di variabili OPD, sia sotto forma di dimensioni OPD, inoltre sarà interessante valutare se e come le dimensioni precedentemente osservate cambieranno nel corso del tempo e come questo cambiamento sarà collegato a esiti differenti e aspetti diversi dell'esito del trattamento (ad esempio, giudizio clinico, valutazione sintomatologica, valutazione nutrizionistica, etc.).

Un ulteriore sviluppo della nostra analisi riguarda l'influenza dei disturbi di personalità sulle dimensioni osservate dei DCA. In particolare siamo andati a osservare la presenza di differenze significative tra le dimensioni OPD riscontrabili (che possono essere riscontrate nei DCA) e i disturbi di personalità. Abbiamo utilizzato inizialmente la diagnostica DSM in Asse II come primo step per arrivare a utilizzare anche qui la diagnostica dimensionale (ad esempio, SWAP- 
200, Westen \& Shedler, 1999a, 1999b) arrivando a ipotizzare l'esistenza di dimensioni a spiegazione singola, cioè non influenzate dal disturbo di personalità ma solo dal DCA, e di dimensioni a spiegazione multipla per cui parte della varianza della dimensione deriva direttamente dall'assetto personologico del paziente.

Concludendo, la diagnosi e l'assessment psicodinamico di cui ci stiamo occupando, risulta essere un ausilio particolarmente utile non solo alla pratica clinica e alla formazione, ma anche alla ricerca sull' origine e sulla comprensione dei disturbi gravi,difficilmente trattabili, aumentando le possibilità di trattamento futuro di tali disturbi e di valutare eventuali caratteristiche evolutivamente significative in tali disturbi.

\section{Valutazione dei fattori terapeutici efficaci nel monitoraggio di trattamenti psicoterapici e psico-sociali e loro implicazioni nei percorsi formativi ${ }^{5}$}

Il problema dell'utilità clinica della conoscenza dei risultati della ricerca può sembrare da un lato automatico e quasi ovvio, dall'altro tutto da dimostrare. Un altro aspetto del problema riguarda le modalità con cui questo passaggio può avvenire nei diversi contesti e in particolare nei Servizi Pubblici di Salute Mentale. Si tratta di valutare, cioè, la fattibilità e l'efficacia di un sistema di formazione basato sull'uso clinico e formativo di alcuni strumenti comunemente usati nella ricerca.

Questo gruppo ha preso spunto dalle conclusioni della 29a Task Force della APA (Norcross, 2001) sui fattori terapeutici di provata o probabile efficacia. La revisione della letteratura empirica sviluppata da questo gruppo, ha evidenziato il ruolo dei fattori terapeutici "aspecifici"

\footnotetext{
${ }^{5}$ Gruppo costituito da E. Fava, A. Ferrari, M. Conte, S.P. Papini, D. Ferrario, D. Taino, M. Tajani, B. Sanna.
} 
e una serie di variabili, relative alla relazione terapeutica, strettamente correlata ai risultati.

Nel costruire il nostro modello (Fava, Ferrario, Sanna, Taino, \& Tajani, in press), denominato Empirically Supported Multi-instrumental Supervision (ESMS), abbiamo da un lato cercato di sviluppare un sistema di valutazione il più semplice possibile, che prendesse in considerazione $\mathrm{i}$ fattori terapeutici di provata o probabile efficacia e dall'altro un metodo per lavorare insieme ai clinici. Il primo passo è stato definire le variabili cliniche da considerare e, successivamente, sono stati scelti gli strumenti più adatti a valutare i fattori terapeutici ritenuti fondamentali. Nella scelta degli strumenti per valutare i trattamenti e l'effetto dell'esperienza formativa abbiamo utilizzato i seguenti criteri:

a) l'esclusione di questionari self report, troppo connessi al funzionamento consapevole e dipendenti dalle aspettative (Williams, 1994; Sitzia \& Wood, 1997). Nei questionari viene infatti considerata la percezione che i soggetti hanno di ciò che sta avvenendo. Nelle analisi delle registrazioni dei colloqui, al contrario, è considerato ciò che avviene realmente nella relazione terapeuta-paziente in modo documentabile;

b) sono stati scelti strumenti che individuano in modo specifico i costrutti che si collegano a fattori terapeutici di documentata efficacia (OPD-2, CCRT-SAI, IVAT-2, SASB, RA).

Nel nostro caso è stato fondamentale considerare le procedure e le modalità del funzionamento del lavoro di gruppo. L'elemento di novità non è stata infatti l'idea di applicare diversi strumenti di ricerca a un singolo caso, bensì la loro applicazione in ambito clinico con obiettivi di formazione e valutazione di qualità.

La modalità di lavoro prevede un incontro preliminare con gli operatori interessati, orientato a comprendere le loro motivazioni, a 
spiegare la natura del progetto e a sviluppare il loro interesse nei confronti dello stesso. Successivamente ci sono stati alcuni incontri di tipo illustrativo sui fattori terapeutici empiricamente supportati, sugli strumenti di valutazione e sul rapporto tra gli uni e gli altri. L'attenzione in questa fase era focalizzata sull'acquisizione del concetto di operazionalizzazione, intesa come abilità di comprendere in modo flessibile la funzione di segnale-indicatore del dato di ricerca rispetto alla realtà clinica.

Nella fase successiva più casi clinici, valutati come problematici, sono stati analizzati da sottogruppi misti di clinici e ricercatori; i risultati sono stati poi discussi prima separatamente e poi nell'insieme. Si è ritenuto, infine, necessario rivalutare il caso clinico dopo un certo periodo di tempo, focalizzando l'attenzione sui processi di cambiamento indotti dalla revisione operazionalizzata.

Brevemente descriveremo le conclusioni e le ipotesi ulteriori a cui il gruppo è giunto:

1. La prima osservazione fondamentale riguarda la possibilità di condurre una discussione clinica fondata su alcuni dati incontrovertibili. Winnicott (1941) osservava che la difficoltà principale nelle discussioni cliniche è il mettersi d'accordo sul significato di ciò che viene osservato. Ciò che viene osservato, nelle discussioni cliniche tradizionali, viene infatti frequentemente "piegato", nel senso di adattato a sostenere ipotesi precostituite. Anche quando si procede attraverso l'analisi di registrati (il che costituisce comunque un significativo passo avanti) è facile che gli eventi osservati vengano interpretati in modo funzionale a un' ipotesi precostituita.

2. Al contrario l'utilizzo di strumenti permette di definire in modo molto chiaro e specifico alcuni eventi (fatti) clinici. Il fatto di poter contare su questa specie di "base solida", empiricamente fondata, non 
impedisce tuttavia ai clinici di integrare queste informazioni con altre che nascono dalla loro esperienza o sensibilità. Questo procedimento non sembra creare problemi di coerenza rispetto alle tecniche e agli approcci teorici dei terapeuti e facilita la comunicazione tra orientamenti diversi in termini propositivi (probabilmente terapeuti rigidamente identificati con il proprio modello non sono disponibili ad entrare in un gruppo di questo tipo).

3. L'applicazione del modello ESMS permette di individuare esplicitamente possibili errori o difetti nella conduzione del trattamento (rotture e assenza di riparazioni nell'alleanza terapeutica, agiti controtransferali, stili relazionali incompatibili, incompletezza o inadeguatezza della comprensione di alcuni aspetti del problema) e di offrire indicazioni per la loro risoluzione. È inoltre possibile, e parimenti importante, il riconoscimento di ciò che viene fatto bene rinforzando in tal modo la fiducia del terapeuta nel trattamento effettuato.

4. Nonostante la marcata laboriosità del lavoro svolto, questo non ha avuto effetti negativi sulla accettabilità e quindi sulla fattibilità del progetto, probabilmente corrispondendo agli interessi dei clinici (è stato per questo scelto un modulo non intensivo). Nessuno dei partecipanti al gruppo, dopo i primi incontri informativi, lo ha abbandonato e vi è stata, inoltre, una richiesta di proseguire anche dopo la conclusione prevista del progetto. È possibile ipotizzare che il training sugli strumenti, che prevede una certa durata e intensità, lasci tracce permanenti nel loro stile di lavoro, maggiori di quelle prodotte da incontri puramente informativi.

5. È necessario che chi conduce il gruppo abbia anche competenze ed esperienza clinica, soprattutto per poter integrare linguaggi in origine diversi anche se ampiamente traducibili e integrabili. Inoltre, il fatto che gli "errori" appaiano in modo piuttosto evidente implica una 
buona coesione del gruppo, quindi che ci sia un ambiente con un basso livello di criticismo per potersi mettere, per quanto possibile, in discussione.

6. Vorremmo infine sottolineare come in questo modo si sviluppi una partecipazione e una riappropriazione da parte dei clinici di quel sapere che sempre più facilmente viene delegato ad agenzie non sempre disinteressate (eufemisticamente) e non sempre realmente competenti, che vorrebbero relegare i clinici nel ruolo di esecutori passivi e acritici. Cosa, questa, che non può non produrre (Brehm \& Brehm, 1981) fenomeni di rifiuto dei percorsi valutativi oppure fenomeni di dipendenza passiva dalle informazioni, con conseguente ulteriore burocratizzazione dei servizi, diminuzione delle competenza clinica e della qualità dei trattamenti.

L'ulteriore sviluppo di questa esperienza, già in atto in altre strutture socio-sanitarie, implica la valutazione dell'efficacia di questo tipo di percorso formativo. Date le motivazioni precedentemente esposte riguardo i limiti dei questionari autosomministrati in questo tipo di lavoro l'ipotesi di lavoro che seguiremo riguarda lo sviluppo di vignette cliniche da sottoporre ai partecipanti, e a un gruppo di controllo, prima e dopo la formazione, che saranno utilizzate come partenza per la misurazione della formazione acquisita.

\section{Bibliografia}

Alpher, V.C. (1991). Interpersonal process in psychotherapy: application to a case study of conflict in the therapeutic relationship. Psychotherapy, 28, 550-562.

American Psychiatric Association (2001). Diagnostic and Statistical Manual of Mental Disorders (4rd. ed. Text Revision). American Psychiatric Association: Washington DC (Tr. it. Manuale diagnostico e statistico dei disturbi mentali (4rd. ed. Text Revision), Masson, Milano 2004. 
Ricerca in Psicoterapia / Research in Psychotherapy 2010; 2 (13): 53-77 http://www.researchinpsychotherapy.net

American Psychiatric Association, DSM-5 Development. Available from http://www.dsm5.org/ProposedRevisions/Pages/RationaleforProposingFiv eSpecificPersonalityDisorderTypes.aspx.

Ba, G., Fava, E., \& Carta, I. (1992). The Training of the Psychiatrist in Relation to the New Trends in Psychiatry. Psychotherapy and Psychosomatic, 57, 8387.

BCPSG (2008). Forms of relational meaning: Issues in the relations between the implicit and reflective-verbal domain. Psychoanalytic Dialogues, 18, $125-148$.

Benjamin, L.S. (1974). Structural Analysis of Social Behavior. Psychological Review, 81, 392-425.

Benjamin, L.S. (1993). Interpersonal Diagnosis and Treatment of Personality Disorders. New York: Guiford Press.

Benjamin, L.S. (2004). Terapia ricostruttiva interpersonale. Promuovere il cambiamento in coloro che non reagiscono. Roma: Las.

Benjamin, L.S. (2005). Interpersonal theory of personality disorders: The Structural Analysis of Social Behavior and interpersonal reconstructive therapy. In M. Lenzenweger \& J. Clarkin (Eds.), Major Theories of Personality, Second Edition. New York: Guilford Press.

Beutler, L.E., Clarkin, J.F., \& Bongar, B. (2000). Guidelines for the systematic treatment of the depressed patient. New York: Oxford University Press.

Beutler, L.E., Harwood, T.M., Michelson, A., Song, X., \& Holman, J.(2011, in session). Resistance/Reactance Level. Journal of Clinical Psychology, 67(2), $1-10$.

Beutler, L.E., Moleiro, C., \& Talebi, H. (2001). Resistance in psychotherapy: What conclusions are supported by research? Journal of Clinical Psychology, 58(2), 207-217.

Beutler, L.E., Rocco, F., Moleiro, C.M., \& Talebi, H. (2001). Resistance. Psychotherapy, 38(4), 431-436.

Brehm, S.S., \& Brehm, J.W. (1981). Psychological Reactance: a Theory of Freedom and Control. New York: Wiley.

Capelli, L., Fava, E., Taglietti, S., Aliprandi, M., Arduini, L., Freni, S., Schadee, H., \& Vigorelli, M. (2005). Relazione interpersonale ed esiti terapeutici: applicazioni del metodo SASB. Ricerca in Psicoterapia, 8, 195-237.

Castonguay, L.G., \& Beutler, L.E. (2005). Principles of therapeutic change that work. New York: Oxford University Press.

Conte, M., Ferrari, A., Fava, E., Papini, S.P., Zuglian, P., Tajani, M., Fiorina, L., Magni, M., Maramieri, L., Primerano, L., \& Freni, S., (2007). Il progetto OPD-Milano: studio di attendibilità tra giudici nella versione italiana. Ricerca in Psicoterapia, 10(1), 11-25.

Fava, E. (1995). Evoluzione del trattamento di un adolescente psicotico. Un'applicazione del metodo CCRT di Luborsky. Adolescenza, 6(1), 33-47. 
Ricerca in Psicoterapia / Research in Psychotherapy 2010; 2 (13): 53-77 http://www.researchinpsychotherapy.net

Fava, E. (2004). Dalla valutazione degli esiti alla competenza a curare: il percorso della ricerca empirica in psicoterapia. Ricerca Psicoanalitica, 15(1), 41-56.

Fava, E., Ferrari, A., Masserini, C., Camarda, I., \& Freni, S. (2002). Esiti e predittori di esiti nelle psicoterapie psicoanaliticamente orientate. Uno studio di efficacia reale in un contesto tipico di trattamento. Ricerca in Psicoterapia, 5(3), 153-185.

Fava, E., Ferrario, D., Sanna, B., Taino, D., \& Tajani, M. (in press). Multinstrumental assessment of psychotherapeutic and integrated interventions quality and clinical staff training. Epidemiologia e psichiatria sociale.

Fava, E., \& Masserini, C. (2002). Efficacia delle psicoterapie nel servizio pubblico. Il contributo della ricerca valutativa alla clinica. Milano: Franco Angeli.

Fava, E., \& Masserini, C. (2006). Psicoterapia nei servizi pubblici. In N. Dazzi, V. Lingiardi, \& A. Colli (Eds.), La ricerca in psicoterapia. Modelli e strumenti (pp. 171-187). Milano: Raffaello Cortina.

Fava, E., Masserini, C., Borghetti, S., Camarda, P., Fontolan, M., \& Duca, P. (2001). Le interruzioni non concordate di trattamento in un servizio di psicoterapia: frequenza, relazioni con gli esiti e variabili con possibile significato predittivo. Rivista Sperimentale di Freniatria, 75(1), 23-36.

Fava, E., Pazzi, E., Arduini, L., Masserini, C., Lammoglia, M., Lomazzi, L., Landra, S., Pazzaglia P., \& Carta, I. (2000). The Effects of psychotherapies: a study on patients perception of results in an Italian public setting. International Journal of Social Psychiatry, 46(4),290-305.

Fava, E., \& Vigorelli, M. (2006). La valutazione del processo terapeutico attraverso un modello circomplesso: la SASB di Lorna Benjamin. In N. Dazzi, V. Lingiardi, \& A. Colli (Eds.), La ricerca in psicoterapia. Modelli e strumenti (pp. 691-711). Milano: Raffaello Cortina.

Fava, E., Zuglian, P., Taino, D., \& Di Genova, C. (2009). La personalizzazione dei trattamenti psicoterapeutici ne disturbi dell'umore. Psichiatria di Comunità, 8(2), 96-105.

Ferrari, A., Pinzi, C., Camarda, P., \& Roustayan, C. (2005). Valutazione a lungo termine dell'effectiveness della psicoterapia supportivo-espressiva in un contesto pubblico: esito, follow-up e predittori. Giornale Italiano Di Psicopatologia, 11, 32-37.

Ferrari, A., Papini, S.P., Zuglian, P., Conte, M., Fava, E., Tajani, M., Fiorina, L., Magni, M., Maramieri, L., Primerano, L., \& Freni, S. (2007). Il progetto OPD-Milano (Diagnosi Psicodinamica Operazionalizzata): nuove configurazioni diagnostiche clinicamente significative. Ricerca in Psicoterapia, 10(1), 27-46.

Fossi, G. (2003). Una proposta evoluzionista per la psicoanalisi. Milano: Franco Angeli Editore.

Freni, S., Azzone, P., Fava, E., Gaburri, L., \& Gigli, G. (1998). La scala di valutazione salute-malattia (Health- Sickness- Rating- Scale, HSRS): 
Ricerca in Psicoterapia / Research in Psychotherapy 2010; 2 (13): 53-77 http://www.researchinpsychotherapy.net

caratteristiche psicometriche e potenzialità di utilizzo della versione italiana. Ricerca in Psicoterapia, 1(3), 278-286.

Gelso, C.J. (2002). Real relationship: The something more of psychotherapy. Journal of Contemporary Psychotherapy, 32, 35-41.

Gelso, C.J. (2004). A theory of the real relationhip in psychotherapy. Paper presented at the International Conference of the SPR Italy - Society of Psychotherapy Research, Rome.

Gelso, C.J. (2009). The real relationship in a postmodern world: theorical and empirical exploration. Psychotherapy Research, 19(3), 253-264.

Henry, W.P. (1996). Structural Analysis of social behaviour as a common metric for programmatic psychopathology and psychotheray research. Journal of Consulting and Clinical Psychology, 64(6), 1263-1275.

Henry, W.P., \& Schacht, T.E. (1994). Modeling recurent patterns of interpersonal relationship with Structural Analysis of social behaviour: The SASB-CMP. Psychotherapy Research, 4, 208-221.

Henry, W.P., Schacht, T.E., \& Strupp, H.H. (1986). Structural analysis of social behaviour: application to a study of interpersonal process in differential psychotherapeutic outcome. Journal of Consulting and Clinical Psychology, 54, 27-31.

Henry, W.P., \& Strupp, H.H. (1994). The therapeutic alliance as interpersonal process. In A.O. Horvath \& L.S. Greenberg (Eds.), The Working Alliance: Theory, Research and Practice. New York: Wiley.

Hilliard, R.B., Henry, W.P, \& Strupp, H.H. (2000). An interpersonal model of psychotherapy: Linking patient and therapist developmental history, therapeutic process, and types of outcome. Journal of Consulting and Clinical Psychology, 68(1), 125-133.

Horvath, A.O. (2009). How real is the "real relationship"? Psychotherapy Research, 19(3), 273-277.

Horvath, A.O., \& Greenberg, L.S. (1994). The Working Alliance: Theory, Research and Practice. New York: Wiley.

Lambert, M.J. (2001). Psychotherapy outcome and quality improvement: Introduction to the special section on patient-focused research. Journal of Consulting and Clinical Psychology, 69, 147-149.

Lomazzi, L., Fava, E., Landra, S., D’Angelo, P., Lammoglia, M., Pazzi, E., Calini, P., Arduini, L., Barattini, D., \& Carta, I. (1997). Le psicoterapie nei centri psicosociali della Lombardia: Il punto di vista di psichiatri e psicologi. Epidemiologia e Psichiatria Sociale, 6(2), 184-93.

Luborsky L. (1962). Clinicians' judgments of mental health: a proposed Scale. Archieves of General Psychiatry, 7, 407-419.

Luborsky, L. (1976). Helping Alliances in psychotherapy: the groundwork for a study of their relationship to its outcome. In J. Claghorn (Ed.), Successful psychotherapy. New York: Brunner e Mazel. 
Ricerca in Psicoterapia / Research in Psychotherapy 2010; 2 (13): 53-77 http://www.researchinpsychotherapy.net

Luborsky, L. (1984). Principi di psicoterapia psicoanalitica. Manuale per il trattamento supportivo-espressivo (Ed. it a cura di S. Freni) Bollati Boringhieri, Torino 1989.

Luborsky, L., Crits-Cristoph P. (1990). Understanding Transference. American Psychological Association, Washington, DC (Tr. it. Capire il transfert, Raffaello Cortina, Milano 1992).

Marchesi, L., Vigorelli, M., Schadee, H., Fava, E., \& Capelli, L. (2007). L'analisi interpersonale del processo terapeutico. Applicazione del modello SASB al caso di Katja. In G. Nicolò \& S. Salvatore (Eds.), La ricerca in Psicoterapia. Materiali e prospettive dal contesto italiano. Roma: Carlo Amore, Firera \& Liuzzo Group.

Masserini, C., Fava, E., Arduini, L., Borghetti, S., Calini, P., Corbellini, G., Ferri, A., Fontolan M., Gatta, S., Mazzariol, M., Pazzi, E,. Spoletini, F., \& Pazzaglia, P. (1998). Drop out in psicoterapie psicodinamicamente orientate: Uno studio con il metodo del CCRT. Ricerca in Psicoterapia, 1(1), 61-67.

Nelson, L.E. (2004). Predicting therapist hostility: Therapist introiect measured by the ISQ, and ability to resist hostile process in-session. Dissertation Abstracts International, 64, 8-B.

Norcross, J.C. (2001). Empirically Supported therapy relationships: Summary report of the Division 29 Task Force. Psychotherapy: Theory, Research, Practice, Training, Special issue, 38, 3-497.

OPD Task Force (1996). Diagnosi Psicodinamica Operazionalizzata. Presupposti teorici e applicazioni cliniche (Tr. it. a cura di A. De Coro, Masson, Milano 2002).

OPD Task Force (2006). OPD-2 Diagnosi Psicodinamica Operazionalizzata. Manuale per la diagnosi e la pianificazione del trattamento (Tr. it. a cura di M. Conte, E. Fava, A. Ferrari, S.P. Papini \& P. Zuglian, Franco Angeli, Milano 2009).

Osimo, F., Merlo, A., Arduini, L, Landra, S., Fava, E., Masserini, C., Carta, I., \& Pazzaglia, P. (1998). Achievement of therapeutic objectives scale. Ricerca in Psicoterapia, 1(2), 153-166.

Perry, J.C. (1991). Defense Mechanism Rating Scale. Unpublished manuscript. Cambridge Hospital, Harvard Medical School, Boston.

Rozzi, S., Fava, E., Lazzari, M., Baruhk, L., \& Fontolan, M. (1997). Valutazione clinica degli esiti e scale di valutazione autosomministrate. Rivista Sperimentale di Freniatria, 81(2), 260-270.

Sasso, G. (2009). Psicoanalisi e neuroscienze. Roma: Astrolabio.

Sitzia, J., \& Wood, N. (1997). Patient satisfaction: A review of issues and concepts. Social Science \& Medicine, 45(12), 1829-1843.

Svartberg, M., \& Stiles, T.C. (1991). Comparative effects of short-term psychodynamic psychotherapy: A meta-analysis. Journal of Consulting and Clinical Psychology, 59(5), 704-714. 
Ricerca in Psicoterapia / Research in Psychotherapy 2010; 2 (13): 53-77 http://www.researchinpsychotherapy.net

Thomä, H., \& Kächele, H. (1985). Trattato di terapia psicoanalitica, 2 vol. (Ed. it a cura di S. Freni) Bollati Boringhieri, Torino 1990.

Verga, M.C., Azzone, P., Viganò, D., \& Freni, S. (1999). Approccio empirico al concetto di alleanza in psicoterapia: alleanza di aiuto, di lavoro, terapeutica. Ricerca in Psicoterapia, 2(1-2-3), 34-62.

Von der Lippe, A.L., Monsen, J.T., Rønnestad, M.H., \& Eilertsen, D.E. (2008). Treatment failure in psychotherapy: The pull of hostility. Psychotherapy Research, 18(4), 420-432.

Wallin, D.J. (2007). Attachment in Psychotherapy. New York: Guilford Press.

Westen, D., \& Shedler, J. (1999a). Revising and assessing Axis II, Part 1: Developing a clinically and empirically valid assessment method. American Journal of Psychiatry, 156, 258-272.

Westen, D., \& Shedler, J. (1999b). Revising and assessing Axis II, Part 2: Toward an empirically based and clinically useful classification of personality disorders. American Journal of Psychiatry, 156, 273-285.

Williams, B. (1994). Patient satisfaction: A valid concept? Social Science \& Medicine, 38(4), 509-516.

Winnicott, D.W. (1941). The observation of infants in a set situation. International Journal of Psycho-Analysis, 22, 229-249 (tr. it. L'osservazione dei bambini piccoli in una situazione prefissata. In Dalla pediatria alla psicoanalisi, Martinelli, Firenze 1975).

Zuglian, P., Papini, S.P., Conte, M., Fava, E., \& Ferrari, A. (in press). Diagnosi Psicodinamica Operazionalizzata. Presupposti teorici, descrizione del sistema e applicazioni dello strumento. Psichiatria di comunità.

\begin{abstract}
The article aim is to briefly describe how our workgroup was born and developed and which research lines we've taken over the years. This aim responds to the shared need and intention of a greater collaboration and integration among different Italian workgroups and to the possibility that this could happen beyond the stimulant, but unfortunately sporadic, contacts that can be established during the national or the international meetings. In this way we could create new ways of research projects sharing. We strongly believe that mutual knowledge is the "main street" for the development of new research projects and for the realization of that scientific knowledge for which our Society was born.
\end{abstract}

\title{
Keywords
}

Efficacy and effectiveness, therapeutic relationship, psychodynamic diagnosis, change resistance, dimensional diagnosis, psychotherapeutic training. 